\title{
NUMERICAL MODELLING OF THE RATCHETTING EFFECT UNDER UNIAXIAL AND MULTIAXIAL LOADING CONDITIONS
}

\author{
AbDelhamid Kerkour-El Miad \\ National School of Applied Sciences (ENSAH), Al-Hoceima, and Faculty of Science, Mohamed Premier University, Oujda, \\ Morocco; e-mail: kerkourelmiad@yahoo.fr \\ Mohammed ABBadi \\ Team of Mechanics and Scientific Computing (ENSA), Mohamed Premier University, Oujda, Morocco \\ e-mail:m.abbadi@ump.ma
}

MOHAMED RADI

AFORPA, CFA, Issy-les-Moulineaux, France

e-mail:med_radi@gmail.com

\begin{abstract}
The main objective of the present work is to numerically test the performance of a micromechanical model under stress-controlled cyclic loading conditions. This simplified nonincremental model has a peculiarity of taking into account the grain shape effect and introduces an isotropic hardening variable for each slip system. The model shows an ability to predict accommodation, uni- and multiaxial ratchetting phenomena for complex loading paths. The uniaxial ratchetting is more pronounced for relatively higher mean stresses. Moreover, the evolution of intragranular isotropic hardening, mainly in path 1 , is found to be dependent on both the sliding nature and the increase of the ACSS number in the case of multiaxial ratchetting. Finally, the main advantage of the explored multiscale approach is in its capability to describe local heterogeneities.
\end{abstract}

Keywords: self-consistent model, uni- and multiaxial ratchetting, non-incremental interaction law, ellipsoidal inclusion, elasto-inelasticity

\section{Introduction}

Historically, it was during the 1960s that scientists became concerned about the uniaxial ratchetting at room temperature. Their main source of motivation consisted in a considerable industrial need, namely, in the air transport and nuclear sectors. Progress in this particular issue has gained a considerable impetus over the past two decades. From the experimental point of view, Ruggles and Krempl (1989) performed an investigation on the ratchetting behaviour of AISI 304 stainless steel at RT, 500, 600 and $650^{\circ} \mathrm{C}$. They observed that the cumulative distortion ratchetting was negligible at high temperatures but large enough for lower temperatures. At RT, the ratchetting strain was found to depend on the loading rate. This characteristic feature was initiated by Yochida et al. (1988). Indeed, those latter reported that this behaviour might be explained by the fact that the material viscosity was affected by temperature. The finding of Ruggles and Krempl (1989) was supported by Chaboche and Nouailhas (1989). Then, the ratchetting conditions were classified into two types: a quasi-alternated condition and a quasi-repeated condition. It should be noted that the cumulative strain ratchetting under quasi-repeated conditions is considered to be caused by viscous effects while the quasi-alternated condition is mainly associated with the cyclic plastic flow. Using the model developed at ONERA, Chaboche and Nouailhas (1989) and Chaboche and Rousselier (1983) showed that the viscosity-based model provided good predictions of the ratchetting behaviour under quasi-repeated conditions. Based 
on the same conditions, Krempl and Yao (1987) also simulated this phenomenon. However, under quasi-alternated conditions, the ratchetting simulation was found to depend on the applied model and, particularly, on the evolution equation of the used kinematic hardening.

Recently, new formulations based on micromechanical approaches have been developed to model the ratchetting behaviour. The advantage of such methods consists in their natural ability to describe the ratchetting effect without additional parameters to obtain realistic predictions of both the hysteresis loop and the ratchetting curve. In this way, a model developed by Abdul-Latif and Radi (2010), is adopted and tested in the present investigation. Thus, the main results predicted by this model are presented in terms of both uniaxial (1D) and biaxial (2D) ratchetting. In the case of fatigue testing performed under stress-controlled loading, the presence of a non-zero mean stress usually leads to a more complex material response (Fig. 1) according to the applied loading conditions. As a matter of fact, three types of responses may be observed:

- The yield strength is exceeded during the first loading cycle resulting in plastic deformation of the material. However, after a few loading cycles (usually around 10 cycles), the material behaviour may become purely elastic (Fig. 1a): this is called the adaptation.

- After a few cycles (generally a few tens of loading cycles), the behaviour can remain plastic, but after an initial increase of the mean strain level at each stress cycle, a stabilised hysteresis loop is reached (Fig. 1b): this is called the accommodation.

- Even after a large number of cycles, the mean strain level keeps increasing at each stress cycle (Fig. 1c): this is called the progressive deformation or ratchetting effect.

(a)

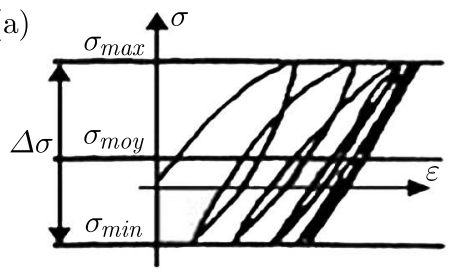

(b)

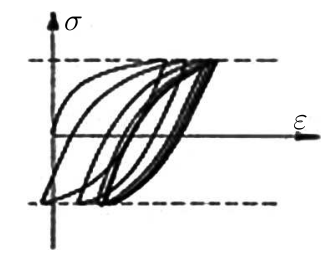

(c)

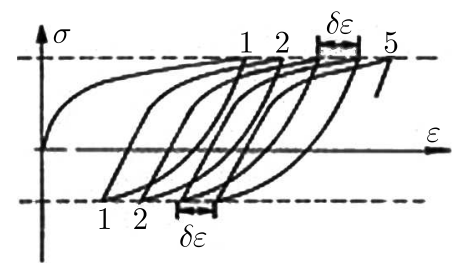

Fig. 1. Schematic representation of low cycle fatigue behaviour in the presence of zero and non-zero mean stresses

The last configuration is prone to take place when the stress range $\Delta \sigma=\sigma_{\max }-\sigma_{\min }$ is greater than $2 k$ in the presence of a non zero mean stress ( $k$ indicates the initial threshold flow). The strain is found to be significant during the first loading cycles before decreasing to reach a constant axial strain increment ranging up to zero in the opposite case (accommodation). To exhibit the progressive deformation, a non zero mean stress during cycling (primary loading) has to be superimposed to a uniaxial or multiaxial cyclic loading (secondary loading). If the cyclic loading is applied in the same direction than the non zero mean stress, the uniaxial ratchetting effect (1D ratchet) takes place. However, when the primary loading direction is different from that of the secondary loading, a multiaxial ratchetting phenomenon occurs. Progressive deformation may then be observed in the loading direction and cyclic hardening (or softening) in the other direction. Two successive ratchetting stages are observed for most of cyclic hardening materials: a transient stage where the strain ratchetting rate per cycle is quite strong followed by a near steady-state where the ratchetting increment $\left(\delta \varepsilon_{p}\right)$ becomes stable with a weaker value than that initially displayed. On the other hand, the ratchetting increment exponentially increases for materials exhibiting cyclic softening behaviour. In overall, the ratchetting effect is not affected by the loading rate at RT. However, the material cyclic hardening plays an important role on the ratchetting behaviour as the kinematic hardening is responsible for this phenomenon. In this way, the present study focuses on two types of ratchetting according to the applied loading: the uniaxial (1D ratchet) and biaxial (2D ratchet) ratchetting. 


\section{Formulation of the model}

The model adopted for this investigation is based on Eshelby's tensor and considers that the elastic behaviour is compressible. This simplified non-incremental model is expressed in the framework of the self-consistent approach of time dependent plasticity. It takes into account the effect of grain shape and introduces an isotropic hardening variable for each slip system. The effect of the kinematic hardening is globally described by the interaction law:

— the slip system level

$$
\begin{aligned}
& \tau^{s}=\boldsymbol{\sigma}^{g}: \mathbf{m}^{s} \quad R^{s}=Q^{s} \sum_{r=1}^{n} H_{r s} q^{r} \\
& \dot{\lambda}^{s}=\left\langle\frac{f^{s}}{K^{s}} z^{z^{s}}=\left\langle\frac{\left|\tau^{s}\right|-R^{s}-k_{0}^{s}}{K^{s}}\right\rangle^{s}\right.
\end{aligned}
$$

— at the granular level

$$
\begin{aligned}
& \left(\overline{\mathcal{I}}^{s-1}+\overline{\mathbf{C}}\right)^{-1}:\left(\dot{\boldsymbol{\sigma}}^{g}-\dot{\boldsymbol{\Sigma}}\right)+\gamma\left(\overline{\mathcal{I}}^{\prime \prime-1}+\overline{\mathbf{A}}^{\prime}\right)^{-1}:\left(\mathbf{s}^{g}-\mathbf{S}\right)=\dot{\boldsymbol{\varepsilon}}^{g}-\dot{\mathbf{E}} \\
& \boldsymbol{\varepsilon}_{e}^{g}=\frac{1+\nu^{g}}{E^{g}} \boldsymbol{\sigma}^{g}-\frac{\nu^{g}}{E^{g}} \operatorname{tr}\left(\boldsymbol{\sigma}^{g}\right) \mathbf{1} \quad \dot{\boldsymbol{\varepsilon}}_{i n}^{g}=\sum_{s=1}^{n} \dot{\gamma}^{s} \mathbf{m}^{s} \\
& \dot{\gamma}^{s}=\dot{\lambda}^{s} \operatorname{sgn}\left(\tau^{s}\right) \quad \boldsymbol{\varepsilon}^{g}=\varepsilon_{e}^{g}+\varepsilon_{i n}^{g}
\end{aligned}
$$

- at the macroscopic level

$$
\begin{aligned}
\dot{\mathbf{E}}_{e}=\sum_{g=1}^{N g} f_{v}^{g} \dot{\boldsymbol{\varepsilon}}_{e}^{g} & \dot{\mathbf{E}}_{i n}=\sum_{g=1}^{N g} f_{v}^{g} \dot{\varepsilon}_{i n}^{g} \\
\dot{\mathbf{E}}=\dot{\mathbf{E}}_{e}+\dot{\mathbf{E}}_{i n} & \dot{\boldsymbol{\Sigma}}=2 \mu \dot{\mathbf{E}}_{e}+\lambda \operatorname{tr}\left(\dot{\mathbf{E}}_{e}\right) \mathbf{1}
\end{aligned}
$$

with

$\tau^{s}$ - resolved shear stress

$\boldsymbol{\sigma}^{g}$ - stress tensor at each grain

$\mathbf{m}^{s}$ - Schmid factor matrix

$R^{s}$ - associated internal stress

$\mathbf{H}=\left\{H_{r s}\right\}$ - interaction matrix of intragranular isotropic hardening

$q^{r}$ - internal "strain" related to isotropic hardening

$Q^{s}$ - isotropic hardening intragranular modulus

$\dot{\lambda}^{s}$ - pseudo-multiplier for each slip system

$K^{s}, Z^{s}$ - constants describing local effect of viscosity of material

$k_{0}^{s}$ - friction stress (critical resolved shear stress)

$\dot{\gamma}^{s}$ - slip rate on slip system

$\overline{\mathcal{I}}^{s}, \overline{\mathcal{I}}^{n}$ - symmetrical fourth rank tensor of elasticity and inelastic interaction fourth rank tensor, respectively

$\overline{\mathbf{C}}$ - global isotropic elasticity tensor

$\gamma$ - material constant (viscoplastic parameter)

$\dot{\boldsymbol{\sigma}}^{g}, \dot{\boldsymbol{\Sigma}}$ - granular and macroscopic Cauchy stress rate tensor, respectively

$\overline{\mathbf{A}}^{\prime}$ - fourth order tensor of macroscopic tangent modulus 
$\mathbf{s}^{g}, \mathbf{S}$ - deviatoric part of granular and macroscopic stress tensors, respectively

$\varepsilon^{g}, \varepsilon_{e}^{g}, \varepsilon_{i n}^{g}$ - total, elastic and inelastic granular strain, respectively

$E, E_{e}, E_{i n}$ - macroscopic total, elastic and inelastic strain, respectively

$E^{g}, \nu^{g}$ - Young's modulus and Poisson's ratio in granular scale, respectively

$f_{v}^{g}$ - volume fraction of grain

$m=m^{g}, l=l^{g}-$ Lamé's constants

1 - second order unit tensor

The dots over strain symbols denote time derivatives (strain rates).

The numerical predictions of this model are explored under stress controlled cyclic loadings with $\gamma$ and $\alpha$ parameters, respectively, equal to $10^{-9}$ and 1.1 (these parameters are obtained after several numerical tests).

\section{Predictions of the model}

\subsection{Uniaxial ratchetting}

Figures below represent predictions of the model for two symmetrical (Fig. 2) and non-symmetrical (Figs. 3 and 4) stress levels. Indeed, Fig. 2 clearly shows an accommodation phenomenon after a certain number of loading cycles. This accommodation effect is also observed

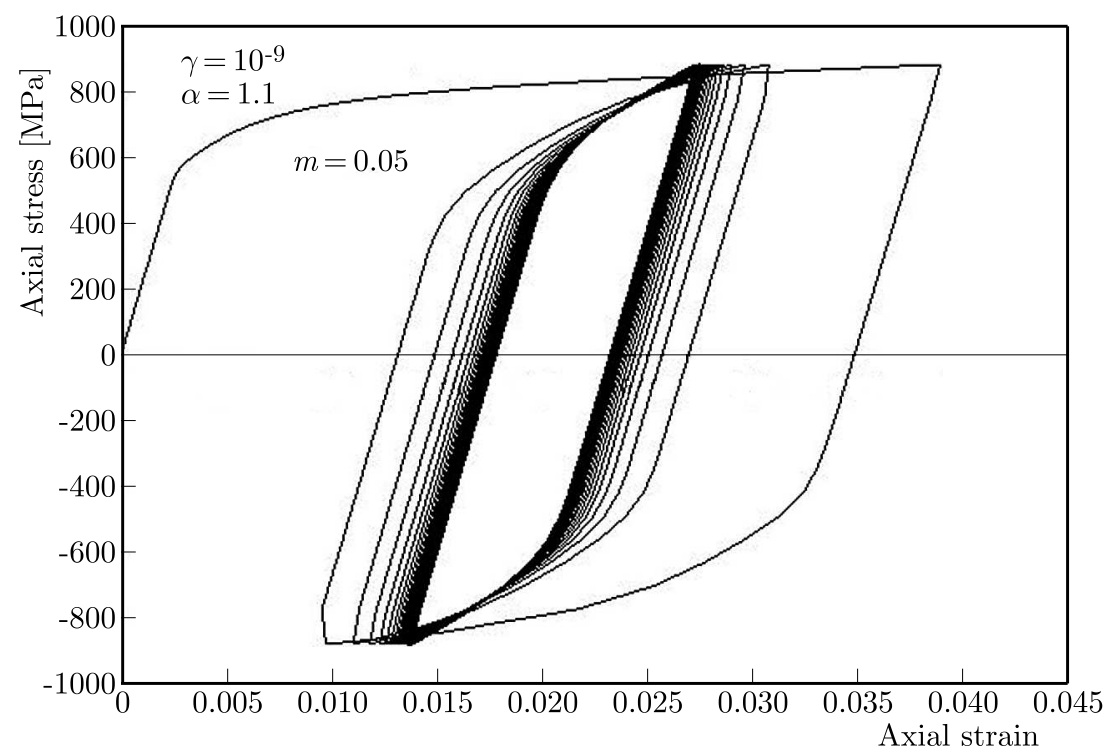

Fig. 2. Response of the model under zero mean stress loading, showing the accommodation phenomenon

when the mean stress is decreased to a value of $\bar{\Sigma}=50 \mathrm{MPa}$ (Fig. 3) instead of $170 \mathrm{MPa}$ corresponding to the apparition of the ratchetting effect (Fig. 4). These findings are in good concordance with the experimental uniaxial ratchetting results obtained by Goodman (1983) on AISI A316 stainless steel at RT. Moreover, they enable, on one hand, to highlight the stabilisation response for low mean stresses (accommodation or adaptation) and, on the other hand, to observe the ratchetting effect for higher mean stresses. Nevertheless, Chaboche et al. (1991) showed that Inconel alloy 718 exhibits the ratchetting effect at $550^{\circ} \mathrm{C}$ irrespective of the mean stress. However, the cumulative progressive deformation remains slow. The influence of the mean stress on the ratchetting phenomenon is depicted in Fig. 5. Indeed, it is observed that the elasto-inelastic behaviour exhibits two stages at $\bar{\Sigma}=0 \mathrm{MPa}$ : the first stage, due to work hardening, 


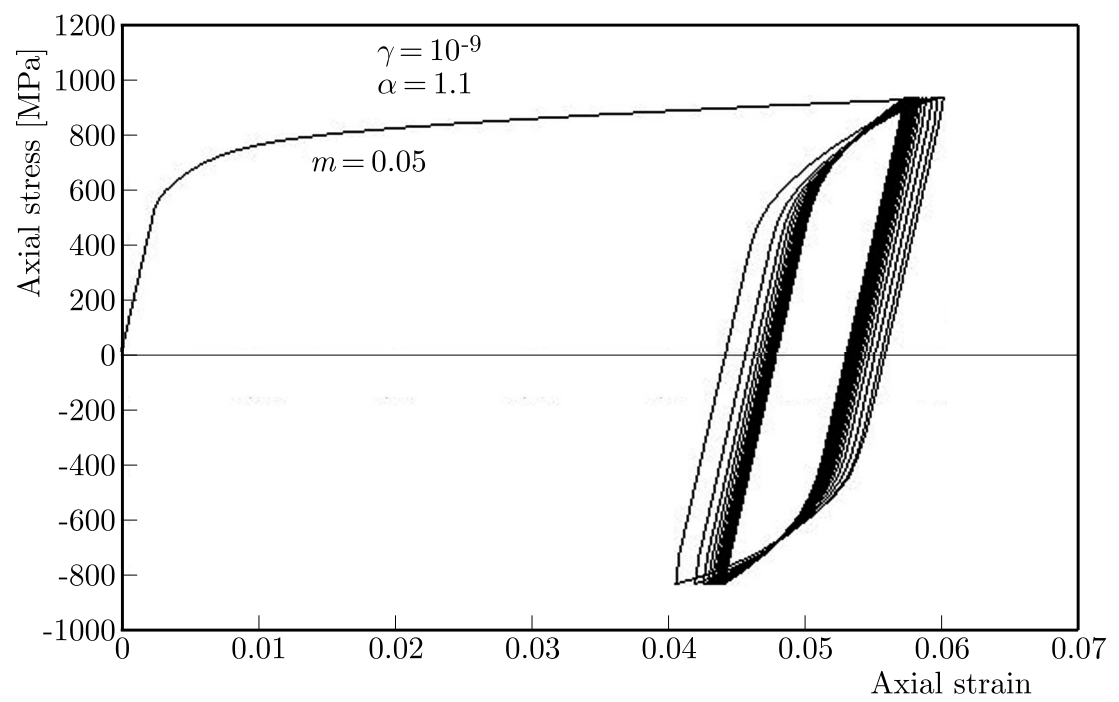

Fig. 3. Response of the model with a non-zero mean stress of $\bar{\Sigma}=50 \mathrm{MPa}$

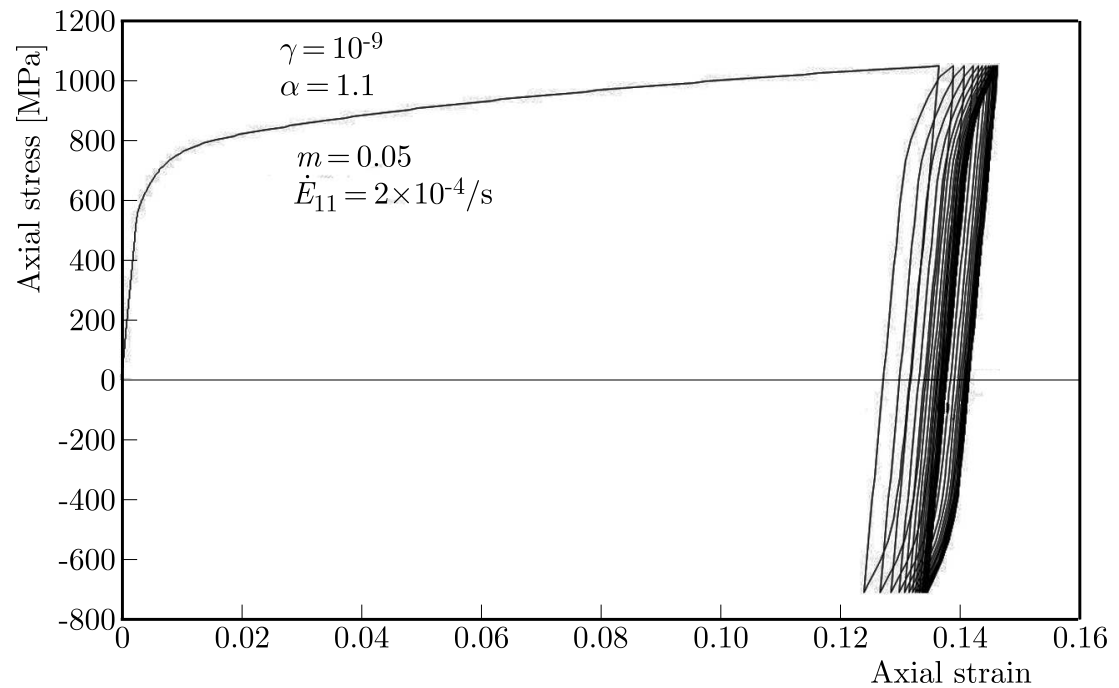

Fig. 4. Response of the model reproducing the phenomenon of uniaxial ratchetting with a non-zero mean stress of $\bar{\Sigma}=170 \mathrm{MPa}$

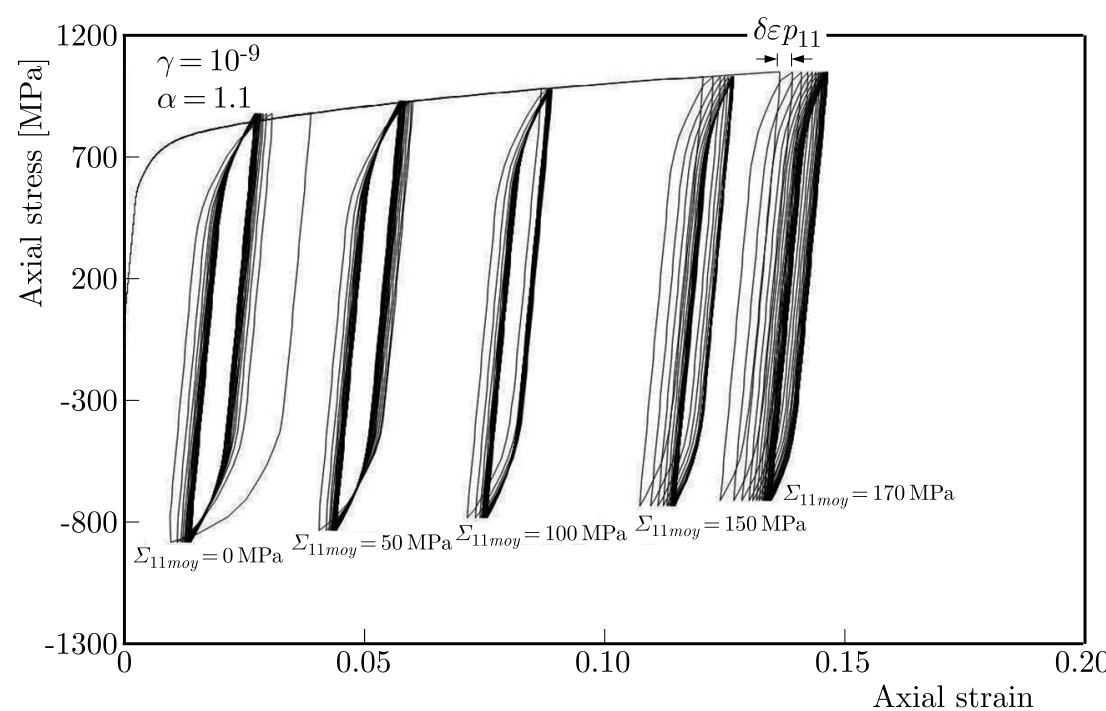

Fig. 5. Effect of the mean stress for many mean stresses varying from $\bar{\Sigma}=0 \mathrm{MPa}$ to $\bar{\Sigma}=170 \mathrm{MPa}$ 
is characterised by the decrease of the inelastic strain while the second exhibits stabilisation of the inelastic strain amplitude, and is known as the accommodation. For $\bar{\Sigma}=50 \mathrm{MPa}$, the ratchetting phenomenon is not observed; however, this stage is transitory between the accommodation and ratchetting. At a mean stress of $100 \mathrm{MPa}$, the ratchetting effect is apparent and becomes more pronounced for higher mean stresses $(\bar{\Sigma}=150 \mathrm{MPa})$. These results suggest that the higher the mean stress, the more significant the increase of the progressive deformation. In view of the above mentioned findings, it can be concluded that the proposed model is capable of reproducing the $1 \mathrm{D}$ ratchetting effect.

\subsection{Multiaxial ratchetting}

As mentioned above, the multiaxial ratchetting arises from superposition of one or several loadings applied to a testing sample in various directions. In the case of $2 \mathrm{D}$ ratchetting, the test consists of a constant amplitude cyclic tension-compression stress combined with a shear cyclic strain in accordance with the work of Portier et al. (2000). Indeed, they performed tests under stress-controlled conditions to reproduce the ratchetting behaviour as depicted in Fig. 6 . The tests were carried out on tubular specimens made of $316 \mathrm{~L}$ stainless steel and COTHAA

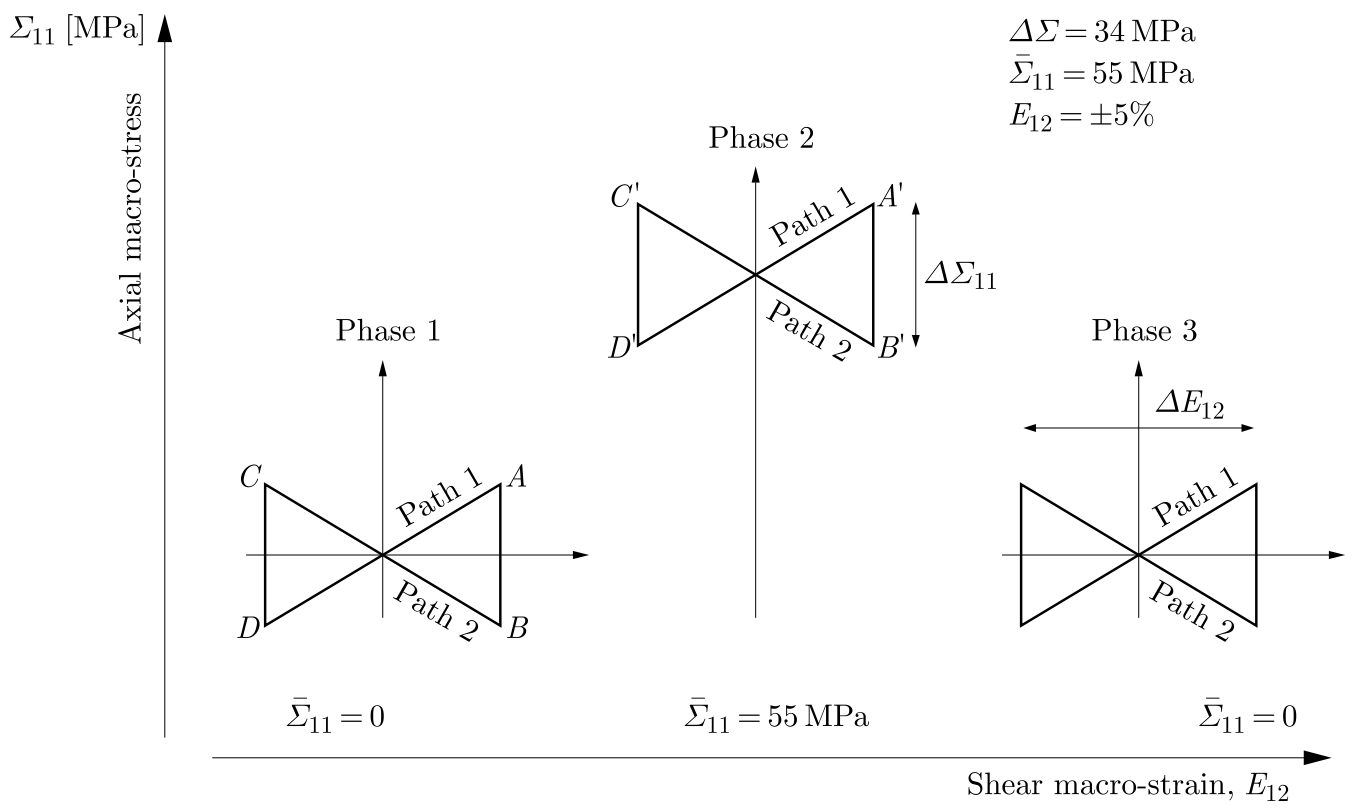

Fig. 6. Multiaxial cyclic loading paths under various mean stresses: bow-tie (path 1) and reverse bow-tie (path 2) (Portier et al., 2000)

steel subjected to tension-shear biaxial loading as follows: bow-tie (path 1) and reverse bow-tie (path 2) cyclic loading situations of three sequences (phases) with different mean stresses $(0,55$ and $0 \mathrm{MPa})$ are respectively imposed with a constant shear strain amplitude of $1 \%$. It should be noted that a constant stress amplitude of $34 \mathrm{MPa}$ is imposed whatever the used path and the applied phase. In addition, path 1 begins with an increase of the axial stress, whereas path 2 begins with a decrease of the axial stress. Various numerical simulations under tension-torsion loading are conducted to test the performance of the explored model. These tests consist in imposing a stress-controlled cyclic tension-compression loading $\bar{\Sigma}_{11}$ and an alternating shear strain $E_{12}$ such that the resulting path describes a bow-tie in $\left(E_{12}, \bar{\Sigma}_{11}\right)$ space. The loading path is described in direction 1 (OABCD) or direction 2 (OBADC), as shown in Fig. 6. Each of the simulations is composed of three successive sequences. The first sequence consists in imposing a bow-tie path with a zero mean stress $\bar{\Sigma}=0 \mathrm{MPa}$ to separate the pure ratchetting effect and the hardening effect of the material. When the shear stress $\bar{\Sigma}_{12}$ is stabilised, the same path but under 
a positive mean stress of $55 \mathrm{MPa}$ is imposed (the second sequence). After a number of cycles, before stabilisation of the ratchetting response, the applied mean stress is removed to observe the ratchetting evanescence. The results obtained by Portier on 316L stainless steel enabled to disparage some models that are known to reflect the classical ratchetting phenomenon such as NLK (Non Linear Kinematics) of Chaboche (1977) and Chaboche and Nouailhas (1989), BCG of Burlet and Cailletaud (1987), OW of Ohno and Wang (1993) and TANA of Tanaka (1994). Vincent et al. (2002), meanwhile, improved the ability of a phenomenological model to reproduce the $2 \mathrm{D}$ ratchetting to palliate the limitations of the kinematics hardening law based-approaches. A comparison between the predictions of the above mentioned models is listed in the work of Bari and Hassan $(2000,2001,2002)$ on the same experimental data. Another approach is proposed by Chache (2004), using the Masing type rule, where the material behaviour is modelled by the parallel association of spring-wiper elements. It should be pointed out that very few results dealing with stress-controlled biaxial loading are found in the literature (Aubin and Degallaix, 2004; Kang et al., 2006; Hassan et al., 2008). However, the majority of biaxial tests reported in the literature is performed under a constant axial stress and cyclic distortion (Portier et al., 2000; Corona et al., 1996) or at a constant internal pressure and cyclic axial strain (Bocher et al., 2001; Corona et al., 1996; Delobelle et al., 1995; Hassan and Kyriakides, 1992; Hassan et al., 1992). Moreover, the model of Abdul-Latif (2004a,b), based on the micromechanical approach, permits good reproduction of the experimental response for three sequences. In overall, the advantage of the micromechanical approaches, as mentioned for the uniaxial ratchetting case, resides in their natural ability to describe this effect without adding any additional parameters.

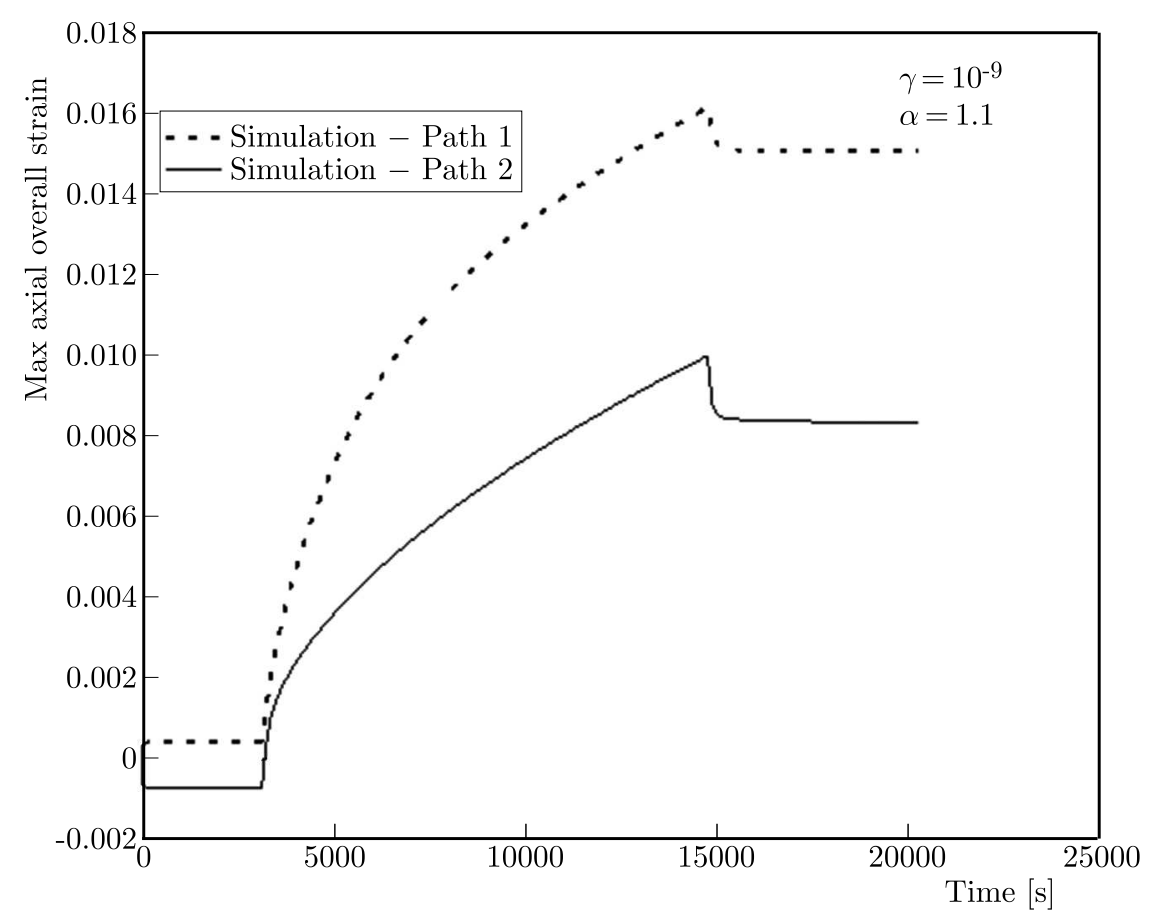

Fig. 7. Model response describing the evolution of the maximum overall strain per cycle by 11 direction during bow-tie (path 1) and reverse bow-tie (path 2)

The numerical results obtained with the model explored in this investigation show its ability to describe the behaviour of the polycristal under complex multiaxial loading (Fig. 7). Indeed, the axial ratchetting is clearly demonstrated for the three sequences irrespective of the loading path. This effect is found to be very small or almost nil during the first sequence. Moreover, loading path 1 contributes to a higher ratchetting effect than path 2 , and the removal of the mean stress during the third sequence results in the evanescence of the ratchet that quickly stabilises. 
The found results enable one to state that the polycristal behaviour is totally dependent on the loading path direction. This finding suggests that the explored model is capable to well reproduce the $2 \mathrm{D}$ ratchetting phenomenon.

A manner to describe the origin of the previous behaviour consists in introducing the local scale variables. For this purpose, the evolution of the intragranular isotropic hardening $R^{s}$ and the shear stress $\tau^{s}$ as a function of the number of cycles is depicted in Figs. 8 and 9 . These figures show that the evolution of $R^{s}$ and $\tau^{s}$ is almost similar during the first sequence regardless of the loading path. However, a change in the local behaviour takes place at the beginning of the second phase, where a mean stress of $55 \mathrm{MPa}$ is imposed. In fact, the evolution of $R^{s}$ is recorded as a typical example for the crystallographic slip system number 11 of the grain number 33 (Fig. 8). During the application of the loading imposed by this phase, an increase of $R^{s}$ is observed with the peculiarity of a rapid increase for path 2 at the onset of loading for a few number of cycles.

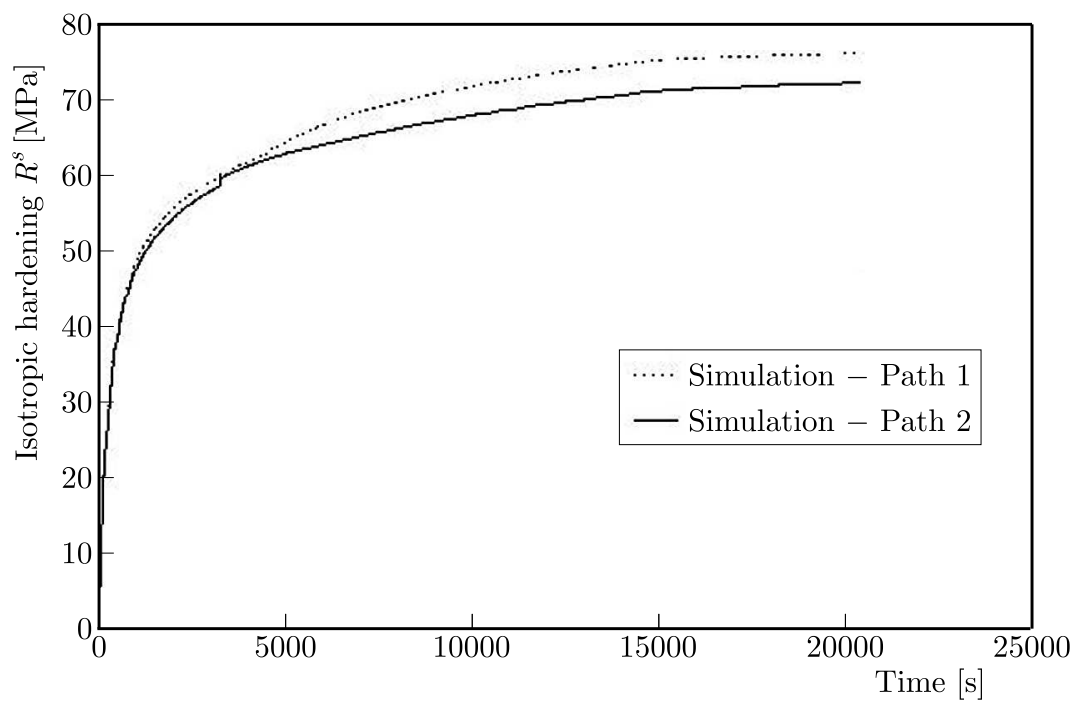

Fig. 8. Evolution of intragranular isotropic hardening system number 11 in the grain number 33 of an aggregate composed of 400 grains

For the remaining loading cycles, the rate of this value decreases, becomes more or less stable and found to be parallely beneath that observed for path 1 . This behaviour leads to gradual evolution of $R^{s}$ during this stage and may be associated with the number of activated crystallographic slip systems per grain ACSS (Fig. 10). The evolution of the average number of slip systems is presented as a global average of the considered aggregate of grains but not only for the grain number 33. Despite this, an objective interpretation may be based on this global evolution. At the onset of the second phase $\bar{\Sigma}_{11}=55 \mathrm{MPa}$, the number of ACSS increases very quickly for path 1 (and decreases abruptly for path 2) and exhibits a slight decrease until the end of this phase. Actually, an increase of the ACSS number has a direct impact on the evolution of $R^{s}$, mainly on its gradual increase observed in path 1 . This evolution is not totally and exclusively governed by the number of ACSS, but also by the nature of sliding, as mentioned in the work of Kerkour-El Miad (2011). In addition, a slight decrease of the rate of evolution of $R^{s}$ is also recorded throughout the third phase (steady state) as shown in (Fig. 8), where $\bar{\Sigma}_{11}$ drops to zero. Figure 10 clearly shows this behaviour through the abrupt decrease of the number of ACSS. In the same way, the evolution of the shear stress is also depicted in Fig. 9 for both paths of loading, on the same slip system that was previously selected (system number 11 of the grain number 33). A major change in the evolution of $\tau^{s}$ is observed in the second phase due to the applied loading (path 1, path 2). Indeed, the evolution of $\tau^{s}$ is found to be soft and progressive for path 1 up to the end of this phase. However, this stress undergoes a brusque shift especially at the onset of this phase for path 2. This behaviour results in an increase of $\tau^{s}$ from 


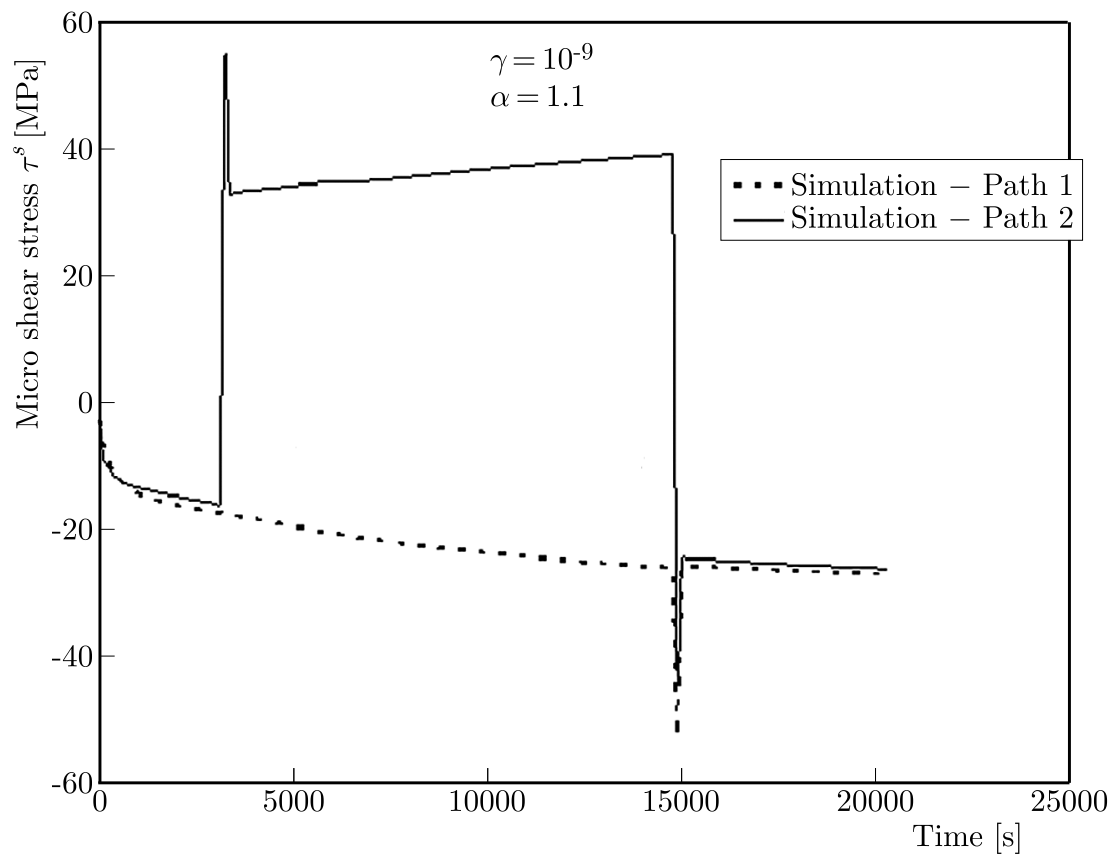

Fig. 9. Variations of shear stress as a function of time for system number 11 in the grain number 33 of an aggregate composed of 400 grains

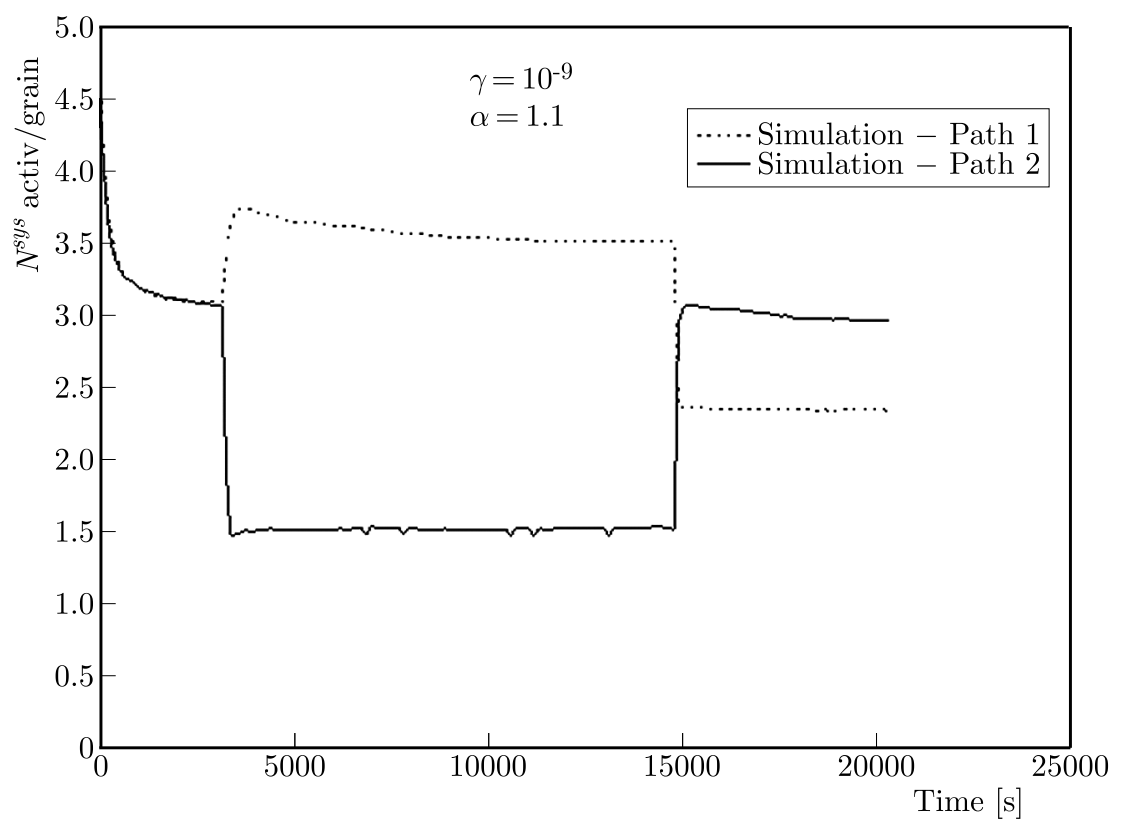

Fig. 10. Evolution of ACSS during the three loading sequences

a negative value of $-16 \mathrm{MPa}$ to a positive value of about $53.4 \mathrm{MPa}$. Afterwards, a very rapid reduction during a short period is noted and leading to a value of $33 \mathrm{MPa}$. This stage is followed by a linear raise until the end of the second phase. For path 1 of the third phase $\left(\bar{\Sigma}_{11}=0 \mathrm{MPa}\right)$, $\tau^{s}$ is clearly affected, but only for a short temporary period by diminution of the mean stress at the global scale before regaining its initial trend. On the other hand, a significant drop of $\tau^{s}$, from 39 to $-42 \mathrm{MPa}$, is noted in path 2 due to the decrease of the mean stress at the global scale. 


\section{Conclusion}

Nowadays, micromechanical approaches have been increasingly adopted by researchers due to their capability to describe local phenomena at the macroscopic scale. The advantage of such approaches lies in the relations of localisation, homogenisation and description of local behaviour. The latter plays an important role in attributing such an ability to micromechanical models. The main goal of the present work is to test the validity of the model used in this investigation under cyclic loading. A particular attention is devoted to the evaluation of the approach prediction in the case of complex loading paths under stress-controlled (uniaxial ratchetting) and strain and stress-controlled conditions (multiaxial ratchetting). The model has proven its ability to reproduce both accommodation and uni- and multiaxial ratchetting phenomena through the performed tests. The uniaxial ratchetting is found to be more pronounced from a mean stress value of $\bar{\Sigma}=150 \mathrm{MPa}$. Moreover, in the case of multiaxial ratchetting, the increase of the ACSS number is found to have a natural consequence on the evolution of $R^{s}$, especially in path 1 . This evolution has turned out to be also dependent on the sliding nature. Finally, the main advantage of the explored multiscale approach is its capability to describe the local heterogeneities.

Acknowledgements

The Authors thank Quartz lab for allowing them to achieve numerical model simulations.

\section{References}

1. Abdul-Latif A., 2004a, A comparison of two self-consistent models to predict the cyclic behavior of polycrystals, ASME Journal of Engineering Materials and Technology, 126, 62.

2. Abdul-Latif A., 2004b, Pertinence of the grains aggregate type on the self-consistent model responses, International Journal of Solids and Structures, 41, 305-322

3. Abdul-Latif A., Radi M., 2010, Modeling of the grain shape effect on the elastic-inelastic behavior of polycrystals with self-consistent scheme, ASME Journal of Engineering Materials and Technology, 132, 1, 011008

4. Aubin V., Degallaix S., 2004, Ratchetting modeling of a duplex stainless steel: model based on yield surface distortion, Proceedings of 7 th International Conference on Biaxial/Multiaxial Fatigue and Fracture, 273-278

5. BARI S., HASSAN T., 2000, Anatomy of coupled constitutive models for ratcheting simulation, International Journal of Plasticity, 16, 381-409

6. BARI S., HASSAN T., 2001, Kinematic hardening rules in uncoupled modeling for multiaxial ratcheting simulation, International Journal of Plasticity, 17, 885-905

7. BARI S., HASSAN T., 2002, An advancement in cyclic plasticity modeling for multiaxial ratcheting simulation, International Journal of Plasticity, 18, 873-894

8. Bocher L., Delobelle P., Robinet P., Feaugas X., 2001, Mechanical and microstructural investigations of an austenitic stainless steel under non-proportional loadings in tension torsioninternal and external pressure, International Journal of Plasticity, 17, 1491-1530

9. Burlet H., Cailletaud G., 1987, Modeling of cyclic plasticity in finite element codes, Proceedings of the 2nd International Conference on Constitutive Laws for Engineering Materials. Theory and Application, C.S. Desai et al. (Edit.), Elsevier, New York, 1157-1164

10. Снавосне J.L., 1977, Viscoplastic constitutive equations for description of cyclic and anisotropic behavior of metals, Bulletin de l'Académie Polonaise des Sciences, Serie des Sciences Technique, $\mathbf{2 5}, 33$

11. Chaвoche J.L., Nouallhas D., 1989, Constitutive modeling of ratchetting effects, ASME Journal of Engineering Materials and Technology, 111, 384-392, 409-416 
12. Chaboche J.L., Nouailhas D., Pacou D., Paulmier P., 1991, Modeling of the cyclic response and ratchetting effects on Inconel-718 alloy, European Journal of Mechanics - A/Solids, 10, 101-121

13. Chaboche J.L., Rousselier G., 1983, On the plastic and viscoplastic constitutive equations, ASME Journal of Pressure Vessel Technology, 105, 153-164

14. Chache M., 2004, Etude de l'écrouissage cyclique des matériaux métalliques et des phénomènes de rochet, Thèse de doctorat, Université J. Fourier, Grenoble-I, France

15. Corona E., Hassan T., Kyriakides S., 1996, On the performance of kinematic hardening rules in predicting a class of biaxial ratchetting histories, International Journal of Plasticity, 12, 117-145

16. Delobelle P., Robinet P., Bocher L., 1995, Experimental study and phenomenological modelization of ratchet under uniaxial and biaxial loading on an austenitic stainless steel, International Journal of Plasticity, 11, 295-330

17. Goodman A.M., 1983, Development of constitutive equations for computer analysis of stainless steel components, 4th International Seminar on Inelastic Analysis and Life Prediction in High Temperature Environment, Chicago

18. Hassan T., Corna E., Kyriakides S., 1992, Ratchetting in cyclic plasticity - Part II: multiaxial behavior, International Journal of Plasticity, 8, 117-146

19. Hassan T., Kyriakides S., 1992, Ratcheting in cyclic plasticity - Part I: uniaxial behavior, International Journal of Plasticity, 8, 91-116

20. Hassan T., Taleb L., Krishna S., 2008, Influence of non-proportionnal loading on ratcheting responses and simulations by two recent cyclic plasticity models, International Journal of Plasticity, 24, 1863-1889

21. Kang G., Kan Q., Zhang J., Sun Y., 2006, Time-dependant ratchetting experiments of SS304 stainless steel, International Journal of Plasticity, 22, 858-894

22. Kerkour-El MiAd A., 2011, Modélisation micromécanique du comportement cyclique des polycristaux sous chargements multiaxiaux à déformation et à contrainte imposées avec l'effet de la forme du grain, Thèse de doctorat, Université Pierre et Marie Curie, Paris, France

23. Krempl E., Yao D., 1987, The viscoplasticity theory based on overstress applied to ratchetting and cyclic hardening, [In:] Low-Cycle Fatigue and Elastoplastic Behavior of Materials, K.T. Rie (Edit.), Elsevier, London, 35-48

24. Ohno N., WANG J.D., 1993, Kinematic hardening rules with critical state of dynamic recovery: Part I: Formulation and basic features for ratcheting behaviour, International Journal of Plasticity, 15, 375-390

25. Portier L., Calloch S., Marquis D., Geyer P., 2000, Ratchetting under tension-torsion loadings: experiments and modelling, International Journal of Plasticity, 16, 3-4, 303-335

26. Ruggles M.B., Krempl E., 1989, The influence of test temperature on the ratchetting behavior of type 304 stainles steel, ASME Journal of Engineering Materials and Technology, 111, 378-383

27. TANAKA E., 1994, A non proportionality parameter and a viscoplastic constitutive model taking into account amplitude dependences and memory effects of isotropic hardening, European Journal of Mechanics - A/Solids, 13, 155

28. Vincent L., Calloch S., Kurtyka T., Marquis D., 2002, An improvement of multiaxial ratchetting via yield surface distorsion, Journal of Engineering Materials and Technology, 124, 4, 402-411, DOI: 10.1115/1.1494450

29. Yoshida F., Kondo J., KikUchi Y., 1988, Visco-plastic behavior of stainless steel SU304 under cyclic loading at room temperature, Transactions of the JSME, A54, 1151-1157 